\title{
La Calidad de las Aguas en el Curso Superior y Medio del Río Traiguén. IX Región-Chile
}

\author{
Nelson R. Rivera ${ }^{1}$, Francisco Encina ${ }^{1,}$ Rodrigo Palma $^{2}$ y Patricia Mejias ${ }^{1}$ \\ (1) Universidad Católica de Temuco, Facultad de Recursos Naturales, Escuela de Ciencias \\ Ambientales, Casilla 15-D, Temuco-Chile (e-maill: nrivera@uct.cl, fencina@uct.cl, pmejias@uct.cl) \\ (2) Servicio Agrícola Ganadero, Bilbao 931, Tercer Piso, Temuco-Chile \\ (e-mail: rodrigopalma@sag.gob.cl)
}

\begin{abstract}
Resumen
En este trabajo se establece la calidad de agua del curso superior y medio del río Traiguén ubicado en la IX Región de la Araucanía en Chile. La caracterización fisicoquímica y microbiológica se efectuó fijando seis estaciones de muestreo, desde un lugar cercano a la naciente del río hasta el límite comunal de la ciudad de Victoria con Traiguén. Los valores mas bajos en los parámetros evaluados, los presentan las aguas de las estaciones 1 y 2 ubicadas fuera del límite urbano, contrariamente a lo que ocurre con las estaciones 3, 4 y 5 que muestran los efectos de los afluentes: planta lácteos, curtiembre y aguas servidas respectivamente. Los resultados de la parte microbiológica señalan que las aguas de ninguna de las estaciones del río pueden emplearse como fuente de abastecimiento para consumo humano por estar sobre los límites fijados por la norma chilena (NCh 409, 1984). Se presentan limitaciones en sus comunidades así como para su uso en regadío, y recreación con contacto directo.
\end{abstract}

Palabras clave: calidad de agua, caracterización fisicoquímica y microbiológica, estaciones de muestreo, medioambiente

\section{Superior and Medium Water Quality in Traiguén River. IX Region-Chile}

\begin{abstract}
The water quality in the upper and medium zone of Traiguén river in the Araucanía region was studied in the present work. Physico-chemical and microbiological parameters were measured at six sample stations in a zone that goes from close to the origin of the river to the limits of the city of Victoria with Traiguén. The influence of the Victoria town on the water quality of Traiguen river generates as consequence low values of studied parameters that were reported in sampling stations 1 and 2 located in the surrounding of Victoria town. A different situation was reported in stations 3, 4 and 5, that show the effects of waste dispose by milky industries, leather industries and waste water respectively. The results of microbiological parameters denoted that the water of all sampled stations can not be used for human use because the parameters are upper than regulations. Also, limitations were denoted in their ecological parameters, and for their use in irrigating and recreational activities.
\end{abstract}

Keywords: water quality, physic-chemical characterization, microbiological characteristics, sampling stations, environment 


\section{INTRODUCCION}

Las características del agua cada día se ven más afectadas por las actividades humanas, las cuales, junto con los características y fenómenos naturales, hacen que sus propiedades fisicoquímicas y microbiológicas varíen notoriamente de un lugar a otro (Gómez et al., 2007). Debido a la alta demanda de agua, es tan importante conocer su calidad para el consumo humano, riego de cultivos, uso industrial, acuicultura y manejo de vida silvestre en general, así como para la recreación y estética (Rivera et al., 2004). Los parámetros de la calidad del agua más frecuentemente utilizados y relevantes para estudios del medio físico, son: Oxígeno disuelto, DBO, sólidos disueltos y en suspensión, compuestos de nitrógeno, fósforo, cloruro, conductividad, $\mathrm{pH}$, dureza, materia orgánica, elementos tóxicos y elementos patógenos (MOPT, 1992).

La calidad del agua no es un término absoluto; dice relación con el uso o actividad a que se destina: calidad para beber, calidad para riego, u otro., por consiguiente, un agua que puede resultar contaminada para cierto uso, puede ser perfectamente aplicable a otro uso; de ahí que se fijen criterios de calidad del agua de acuerdo a los usos (Murgel, 1984). En Chile, la norma legal sobre requisitos de calidad del agua para diferentes usos es la Norma Chilena 1333 (NCh 1333,1978).

La normativa chilena que regula la calidad del agua superficial que puede ser usada como fuente de suministro de agua potable es la Norma Chilena 777 (NCh 777, 1971), que clasifica la calidad del agua, sobre la base de diez parámetros y la Norma Chilena 409 (NCh 409/1, 1984), que establece los requisitos físicos, químicos, radioactivos y bacteriológicos que debe cumplir el agua potable proveniente de cualquier sistema de abastecimiento.

La ciudad de Victoria, ubicada en la Novena Región presenta una población urbana de 23.977 habitantes (INE, 2002). Las industrias ubicadas en Victoria son principalmente: matadero, aserraderos y lácteos (DGA, 2004). Las descargas tanto de riles como aguas servidas no tratadas llegaban al río Traiguén (CONAMA, 1998), sólo en Febrero del 2001 entró en funcionamiento una planta de tratamiento de aguas servidas.

Los residuos líquidos urbanos y domésticos $\left(0,1-0,3 \mathrm{~m}^{3}\right.$ /persona / día) contienen sales minerales, materia orgánica, detergentes, restos de compuestos no biodegradables y microorganismos, que conforman una mezcla de gran complejidad química causando la disminución de oxígeno en las aguas del cuerpo receptor (Doménech, 1994). Herraéz y Rubio (1989), indican que la industria láctea genera nitrógeno 1 a $180(\mathrm{mg} / \mathrm{L})$, fósforo $\left(\mathrm{PO}_{4}\right) 9$ a $210(\mathrm{mg} / \mathrm{L})$, temperatura desde 8 a $45^{\circ} \mathrm{C}$ y cloruros desde 46 a 1930 (mg/L). Los desechos lácteos contienen esencialmente lípidos no emulsificados, lactosa y caseína. La industria de productos lácteos tiene un alto consumo de agua en operaciones de limpieza, calentamiento, enfriamiento, secado, etc., con la consiguiente generación de desechos líquidos que van desde 1 a $20 \mathrm{~m}^{3}$ /ton producción en promedio.

El objetivo de este trabajo fue determinar la calidad fisicoquímica y microbiológica de las aguas en el curso superior y medio del río Traiguén, que permitan construir líneas de base adecuada para los estudios de impacto ambiental y otras determinaciones a nivel gubernamental, empresarial y otros. Para evaluar a futuro los cambios de la calidad de aguas del río Traiguén en la zona urbana de la ciudad de Victoria asociados a la puesta en marcha de la Planta de Tratamiento de aguas servidas, se fijó la estación de muestreo 1 lo más cercana a la naciente del río para ser considerada estación de referencia.

\section{MATERIALES Y MÉTODOS}

El régimen hidrológico de los ríos de la Región de la Araucanía es pluvial. Esta característica está asociada a que la cordillera de los Andes es normalmente baja en altura en esta región, por lo que no se acumula mucha nieve (Rivera et al., 2004). La subcuenca del río Traiguén pertenece a la hoya hidrográfica del río Imperial, limitando al norte con la cuenca del río Biobío y al sur con la del río Toltén, posee una superficie de 10176 ha cuyo uso está distribuido en Agrícola 50,05\%, Forestal $25,8 \%$, presencia de renovales $19,39 \%$, pradera $4,56 \%$ y urbano $0.19 \%$. La comuna de Victoria 
cuenta con una superficie de riego de 101.075 hectáreas, de las cuales 1.400 hectáreas son regadas con aguas del río Traiguén.

Su origen es preandino, a $20 \mathrm{~km}$ al oriente de la ciudad de Victoria, de la unión de los esteros las Mariposas y Collahue y su longitud es de $50.4 \mathrm{Km}$. El río Traiguén es un tributario de orden 3 de la cuenca del río Imperial (1.276.260 ha) y drena una superficie de 10176 ha. El tamaño de la subcuenca es semejante a otras presentes de la depresión intermedia de la zona sur (VIII, IX y X regiones) donde la intensidad de uso es predominantemente agrícola y en los últimos 15 años, se ha visto un importante incremento del uso forestal sobre todo en la VIII región. El relieve es relativamente plano (1 a $5 \%$ ) y el perímetro alcanza los $96 \mathrm{Km}$. Las precipitaciones anuales en la cuenca son del orden de 1500 a $2000 \mathrm{~mm}$ y temperaturas de $23^{\circ} \mathrm{C}$ máxima mes de enero y $-5^{\circ} \mathrm{C}$ mínima mes de Julio. Su caudal medio máximo de $12,56 \mathrm{~m}^{3} / \mathrm{s}$ en invierno (Julio) y de $0.56 \mathrm{~m}^{3} / \mathrm{s}$ en verano (Marzo). El Clima es templado lluvioso, característico de la región centro-sur de Chile.

Se muestreo en tres oportunidades en triplicado durante el mes de Noviembre del año 2000. Se fijaron 6 estaciones de muestreo (Fig.1). Las estaciones 1 y 2 están fuera del límite urbano presentando caudales de 12,24 y 10,13 ( $\left.\mathrm{m}^{3} / \mathrm{s}\right)$ respectivamente, la estación 3 recibe el afluente de una planta lechera, la estación 4 que presenta un caudal de $6,83 \mathrm{~m}^{3} / \mathrm{s}$ recibe el afluente de una curtiembre, la estación 5 que presenta un caudal de $7,82 \mathrm{~m}^{3} / \mathrm{s}$ recibe el afluente urbano (aguas servidas) y la estación 6 ubicada en el límite comunal de Victoria con Traiguén. Se determinó muestrear en Noviembre al considerar que el año anterior fue el mes que presentó el menor caudal medio. Cada estación fue georeferenciadas con un GPS, marca Magellan, Modelo Color Track. El caudal instantáneo fue estimado de acuerdo con la metodología de Linsley (1992) y Harvey (1993). Simultáneamente se midió la velocidad de corriente mediante un Flujómetro marca Global Flow Pro, modelo FP-201, con una sensibilidad de 0,1 m/s.

Los parámetros físico químicos fueron medidos en muestras de superficie $(0-30 \mathrm{~cm})$, la temperatura y el pH fueron medidos con pHmetro Orión modelo 250A, con una sensibilidad de 0,01 unidad, la conductividad, salinidad y sólidos disueltos totales (TDS) fueron medidos con equipo Orión modelo 115 , con una sensibilidad de 0,01 mg/L. La extracción de las muestras de agua se realizó con botella tipo Van Dorn horizontal de $3 \mathrm{~L}$, extrayendo en cada estación 10 litros de agua depositados en bidones plásticos para el análisis de fosfato, amonio, nitrito y nitrato. Para el análisis microbiológico se extrajo agua en botellas de vidrio de $1000 \mathrm{ml}$. Todos los envases fueron sometidos a los protocolos de limpieza y esterilización correspondientes de acuerdo a la Norma Chilena 411/6 (NCh $411 / 6,1999)$ y al Manual de Calidad del laboratorio.

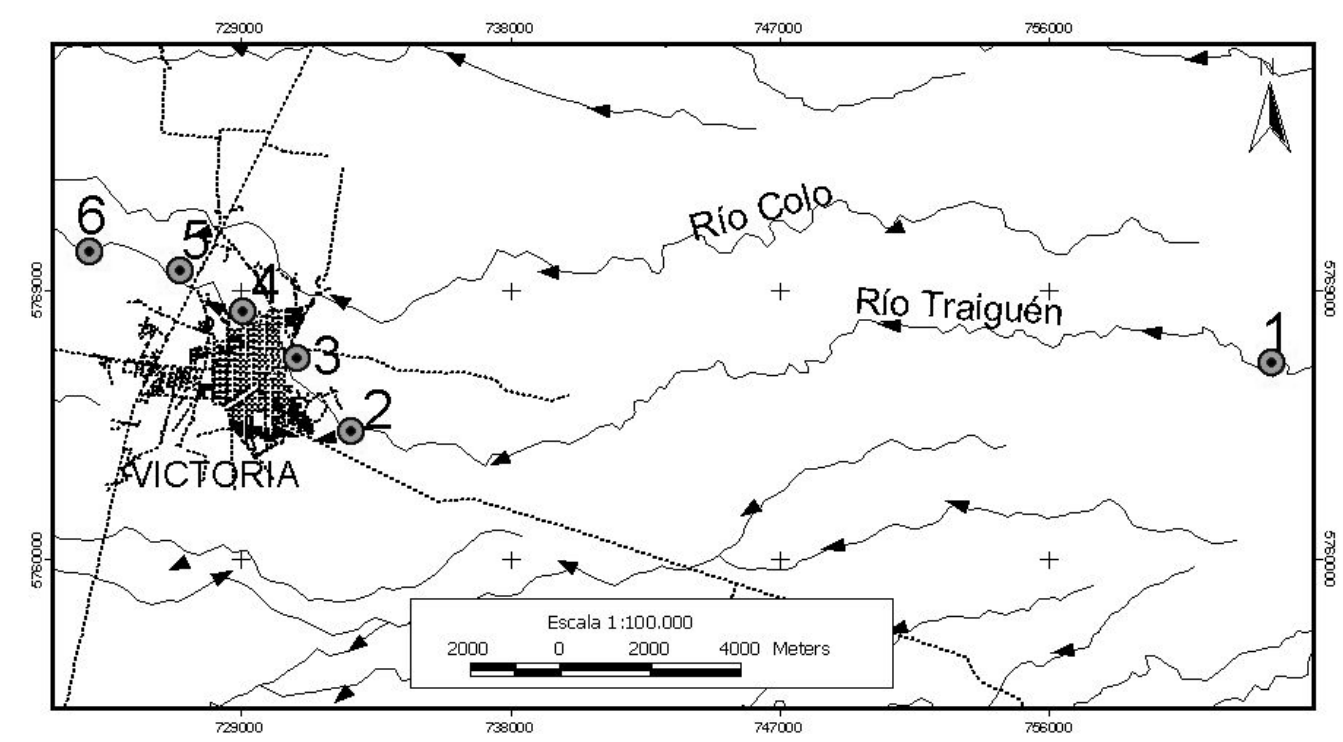

Fig. 1: Ubicación geográfica del río Traiguén (IX Región-Chile) y distribución espacial de las estaciones de muestreo (1.-Mariposas, 2.- Bocatoma ESSAR, 3.- Lechera, 4.- Curtiembre, 5.- Ducto alcantarillado, 6.- Turbina) 
Las muestras de oxígeno disuelto y de $\mathrm{DBO}_{5}$ fueron extraídas en botellas para DBO y llevadas a temperatura controlada al laboratorio. Los parámetros químicos fueron analizados de acuerdo a las Normas Chilenas vigentes y para los análisis microbiológicos se utilizó la técnica del número más probable (NMP) en 100ml. Los laboratorios encargados de la parte analítica, cuentan con un Manual de Calidad que especifica un control interno de calidad que contempla analizar estándares de verificación, duplicados y muestras adicionadas.

La comparación entre estaciones se realizó mediante análisis de varianza previa comprobación de normalidad y homogeneidad de la varianza. Los análisis a posteriori se realizaron mediante Test de Tukey (Zar, 1984). Las relaciones entre las estaciones y los parámetros físico químicos se realizaron mediante el Análisis de Componentes Principales (ACP) de acuerdo a Field et al. (1982) y Hair et al (1998). Esta técnica es esencialmente descriptiva y permite analizar la estructura de correlación entre las variables. El propósito de ACP es encontrar espacios de menores dimensiones en los cuales sea posible observar las relaciones entre los individuos de mejor manera. Para la aplicación de la ACP, se utilizó la matriz de correlación a partir de la Tabla 1, no se realizó rotación de ejes. La selección del número de componentes consideró aquellos ejes con valores propios superiores a 0.8 , otro criterio fue la regla del codo que corresponde a que una vez ordenados los valores propios en forma descendente se escogen los primeros, antes de cambiar la pendiente (Hair et al., 1998). Para los análisis se utilizaron los programas Excel 2003, Statgraphics (5.0) y XLSTAT para Windows.

\section{RESULTADOS Y DISCUSIÓN}

Los resultados promedios de los parámetros fisicoquímicos y microbiológicos analizados se presentan en la tabla 1. La temperatura del agua es un parámetro importante, ya que de ella dependen otros parámetros, por ejemplo la conductividad. Seoanez (1995), menciona que esto se debe a que dependiendo de la temperatura aumenta o disminuye la solubilidad de las sales y en especial la de los gases, modificándose por tanto la concentración de las especies iónicas presentes. Las mediciones realizadas en las Estaciones de muestreo de las aguas del río Traiguén muestran los valores más bajos en las estaciones 1 y 2 , debido a que se encuentran ubicadas fuera del límite urbano, en la zona ritrónica del río con mayor pendiente y velocidad.

Las temperaturas presentan un mínimo de $11,7{ }^{\circ} \mathrm{C}$ en la estación 1 y un máximo de $20,3{ }^{\circ} \mathrm{C}$ en la estación 5, cumpliendo con lo establecido en la Norma Chilena 1333 (NCh 1333, 1978) para recreación con contacto directo. Para riego la Norma Chilena no considera la temperatura entre sus parámetros a controlar.

El pH presentó su máximo valor de 9.7 en la Estación 3, lo que se debe a que en esta estación existe un efluente de una industria lechera, con $\mathrm{pH}$ básico lo que ocasiona un aumento en el $\mathrm{pH}$ del cuerpo receptor. El mínimo fue 6.5 en la Estación 6, no existiendo diferencias significativas entre las Estaciones 1 y 2 y las Estaciones 4 y 5, presentando un valor $p<0.05$. Considerando los requisitos para agua de riego, según la Norma Chilena 1333 (NCh, 1978), estas deben tener un pH entre 5.5 y 9.0, indicando que las aguas del río son aptas para riego, excepto la Estación 3 que supera la norma en 0.7 unidades en promedio de $\mathrm{pH}$. Los requisitos de $\mathrm{pH}$ para aguas destinadas a recreación y estética, según la Norma (NCh 1333, 1978), deben estar entre 6.5 y 8.3 y en ningún caso menor de 5.0 o mayor de 9.0 , de acuerdo a esto el río Traiguén estaría fuera del rango de pH permitido, ya que las Estaciones 1, 2 y 3 superan los rangos de la Norma Chilena. El requisito para la vida acuática es de 6.0 a 9.0 unidades, por lo que la estación 2 y 3 no cumplen con los requisitos propuestos por la Norma (NCh 1333, 1978).

La conductividad presentó su máximo valor en la Estación 5 con $0.15 \mu \mathrm{S} / \mathrm{cm}^{2}$ y el mínimo se encontró en la Estación 1 con $0.03 \mu \mathrm{S} / \mathrm{cm}^{2}$, existen diferencias significativas entre todas las estaciones, con un $p<0.05$. La conductividad del agua da una estimación acerca de la concentración aproximada de las sales minerales presentes, lo que es de utilidad en cultivos agrícolas (Seoanez, 1995). La Norma Chilena 1333 (NCh 1333, 1978) sólo considera la conductividad para el uso de riego con un rango de 0.75 a $7.5 \mu \mathrm{S} / \mathrm{cm}^{2}$. Las aguas del río Traiguén, en todas sus estaciones, se encuentran dentro del límite establecido por la Norma Chilena. 
En cuanto a la salinidad, esta no se considera en las Normas Chilenas (NCh 777/71, NCh 1333/78 y NCh 409/84). Para las Naciones Unidas (CONAMA, 1997) este parámetro es requisito para el agua de riego, con un rango de $<0.0007$ a $>0.003 \mathrm{mg} / \mathrm{l}$. Las aguas del río Traiguén mostraron variaciones desde 0 a $0.1 \mathrm{mg} / \mathrm{l}$, lo que indicaría que las Estaciones 5 y 6 están por sobre el límite establecido por las Naciones Unidas.

Los sólidos disueltos totales presentaron su máximo valor en la Estación 5 con 80 mg/l y el mínimo en la Estación 1 con $13.8 \mathrm{mg} / \mathrm{L}$, existiendo diferencia significativa entre todas las estaciones, con un valor $p<0.05$. La Norma Chilena 1333 (NCh, 1978) considera como requisito del agua para riego el rango de 500 a $5000 \mathrm{mg} / \mathrm{L}$ para sólidos disueltos totales. Las aguas del río, en todas las estaciones, se encuentran dentro de este rango (Tabla 1). Para captación de agua para consumo humano los sólidos disueltos totales sólo son considerados por la OMS (2004), todas las estaciones se encuentran dentro del rango. Para los sólidos en suspensión la Norma Chilena 1333 (NCh 1333, 1978), considera como requisito del agua para uso de vida acuática la ausencia de sólidos en suspensión. Las Estaciones presentaron variaciones desde 0.0006 a $0.011 \mathrm{mg} / \mathrm{L}$, estando por sobre la Norma (Tabla 1).

El oxígeno disuelto presentó su máximo valor en la Estación 1 con $10.31 \mathrm{mg} / \mathrm{L}$ y el mínimo en la Estación 6 con $1.48 \mathrm{mg} / \mathrm{L}$, existiendo diferencia significativa entre las Estaciones 2, 3 y 4 y entre las Estaciones 5 y 6 , con un valor $\mathrm{p}<0.05$. Las estaciones $5(4,52 \mathrm{mg} / \mathrm{L})$ y $6(1,48 \mathrm{mg} / \mathrm{L})$ presentan valores menores a los $5 \mathrm{mg} / \mathrm{L}$ para la protección de la vida acuática establecida en la Norma Chilena 1333 (NCh 1333, 1978). Para el río Traiguén el promedio de oxígeno disuelto fue de $6.74 \mathrm{mg} / \mathrm{L}$, siendo una concentración baja, ya que la saturación de oxígeno a la temperatura del agua de $17.1^{\circ} \mathrm{C}$ es de $9.62 \mathrm{mg} / \mathrm{L}$.

Para fosfato, el río presentó su máximo valor en la Estación 5 y fue de $5.61 \mathrm{mg} / \mathrm{l}$ y el mínimo valor en la Estación 1 con $0.06 \mathrm{mg} / \mathrm{l}$, existiendo diferencia significativa entre las Estaciones 1 y 2, con un valor de $\mathrm{p}<0.05$. El ión fosfato $\left(\mathrm{PO}_{4}\right)$ es uno de los factores limitantes en el crecimiento vegetal (Rivera et al., 2004). Si bien las Normas Chilenas no consideran el fosfato en los parámetros para diferentes usos, hubo apreciables diferencias entre las primeras cuatro Estaciones y las Estaciones 5 y 6 , lo cual se puede explicar por el impacto del afluente urbano (aguas servidas) en esa zona. (Tabla 1).

Nitrógeno amoniacal, nitritos y nitratos, son indicadores de contaminación orgánica de aguas superficiales. Para el nitrógeno amoniacal, el río presentó su máximo valor en la Estación 5 con 7,57 $\mathrm{mg} / \mathrm{L}$ y el mínimo valor en la Estación 1 con $0.031 \mathrm{mg} / \mathrm{L}$, no existiendo diferencias significativas entre las Estaciones 1, 2 y 4, con un valor $p<0.05$. El límite máximo fijado por la Norma Chilena 409 (NCh 409, 1984), es de 0.25 mg/L, las Estaciones 5 y 6 están por sobre el límite máximo.

En cuanto a nitrito el río presentó su máximo valor en la Estación 3 con $0.057 \mathrm{mg} / \mathrm{l}$ y el mínimo valor en la Estación 1 con $0.012 \mathrm{mg} / \mathrm{l}$, no existiendo diferencias significativas entre las estaciones 2 y 6 , presentado un valor $\mathrm{p}<0.05$. La Norma Chilena 409 (NCh 409, 1984), considera un valor máximo para los nitritos de $1 \mathrm{mg} / \mathrm{L}$. Estando las aguas del río, en todas las estaciones, dentro del rango descrito por la normativa vigente.

Para nitrato el río presentó su máximo valor en la Estación 5 con $0.9 \mathrm{mg} / \mathrm{L}$ y el mínimo valor en la Estación 1 con $0.2 \mathrm{mg} / \mathrm{L}$ (Tabla 1), no existiendo diferencias significativas entre las estaciones 2 y 6 , con un $\mathrm{p}<0.05$. El requisito "para captación de agua para consumo humano" según la Norma Chilena 409 (NCh 409, 1984), es de 10 mg/L y las aguas del río Traiguén están dentro del rango que ésta exige. El Nitrógeno Amoniacal, los Nitritos y el Nitrato, no son considerados en la Norma Chilena 1333 (NCh 1333, 1978), para agua destinada a riego, se debe considerara su importancia como fertilizante, en dosis elevadas genera efectos adversos.

Para sulfato el río presentó su máximo valor en la Estación 5 con $0.976 \mathrm{mg} / \mathrm{L}$ y el mínimo valor en la Estación 1 con $0.019 \mathrm{mg} / \mathrm{L}$, existe diferencia significativa entre todas las estaciones (valor $\mathrm{p}<0.05$ ). Los sulfatos están ampliamente distribuidos en la naturaleza y en aguas naturales están presentes en concentraciones variables. Las Estaciones 5 y 6 presentaron los valores más altos en sulfato (Tabla 1). La Norma Chilena 409 (NCh 409, 1984), presenta un valor máximo de 250 mg/L y para 
captación de agua para riego en la Norma Chilena 1333 (1978), el valor máximo es de 250 mg/L. Las aguas del río Traiguén se encuentran dentro de los rangos máximos establecidos por ambas normas.

Tabla 1: Análisis Fisicoquímico de las aguas del río Traiguén, IX Región de Chile.

\begin{tabular}{|c|c|c|c|c|c|c|}
\hline & \multicolumn{6}{|c|}{ Estaciones } \\
\hline Parámetros & 1 & 2 & 3 & 4 & 5 & 6 \\
\hline $\mathrm{T}^{\circ} \mathrm{C}$ & $11.7 \pm 0,10$ & $14.8 \pm 0,15$ & $17.6 \pm 0,15$ & $19.9+0,0$ & $20.3+0,35$ & $18.1 \pm 0,2$ \\
\hline $\mathrm{pH}$ & $8.9+0,0$ & $9.2 \pm 0,2$ & $9.7 \pm 0,12$ & $7.2 \pm 0,06$ & $6.9 \pm 0,0$ & $6.5 \pm 0,06$ \\
\hline $\begin{array}{l}\text { Cond. } \\
(\mu \mathrm{s} / \mathrm{cm})\end{array}$ & $0.03 \pm 0,00$ & $0.05 \pm 0,00$ & $0.07 \pm 0,00$ & $0.05 \pm 0,00$ & $0.15 \pm 0,00$ & $0.14 \pm 0,00$ \\
\hline TDS (mg/L) & $13.8 \pm 0,0$ & $16.9 \pm 0,0$ & $28.1 \pm 0,0$ & $26.2 \pm 0,0$ & $80.0 \pm 0,0$ & $70.9 \pm 0,0$ \\
\hline Sal $(g / L)$ & 0 & 0 & 0 & 0 & $0.1 \pm 0,0$ & $0.1 \pm 0,0$ \\
\hline $\begin{array}{l}\text { Oxígeno } \\
\text { (mg/L) }\end{array}$ & $10.31 \pm 0,01$ & $8.49 \pm 0,00$ & $8.28 \pm 0,08$ & $7.39 \pm 0,01$ & $4.52 \pm 1,48$ & $1.48 \pm 0,01$ \\
\hline DBO (mg/L) & $2,67 \pm 0,023$ & $0.48+0,280$ & $3.38+0,007$ & $7.06+0,293$ & $4.62+0,08$ & $1.53+0,0$ \\
\hline Fosfato $(\mathrm{mg} / \mathrm{L})$ & $0.031 \pm 0,001$ & $0.038+0,002$ & $0.221 \pm 0,001$ & $0.156 \pm 0,002$ & $5.61 \pm 0,03$ & $2.04 \pm 0,02$ \\
\hline $\begin{array}{l}\text { N-amoniacal } \\
(\mathrm{mg} / \mathrm{L})\end{array}$ & $0.06 \pm 0,01$ & $0.11 \pm 0,01$ & $0.21 \pm 0,012$ & $0.18 \pm 0,012$ & $7.57 \pm 0,06$ & $4.33 \pm 0,12$ \\
\hline Nitrato(mg/L) & $0.20 \pm 0,00$ & $0.4 \pm 0,0$ & $0.70 \pm 0,0$ & $0.57 \pm 0,06$ & $0.9+0,0$ & $0.33+0,06$ \\
\hline Nitrito(mg/L) & $\begin{array}{c}0.012 \\
+0,000\end{array}$ & $\begin{array}{c}0.018 \\
+0,000\end{array}$ & $\begin{array}{c}0.057 \\
+0,001\end{array}$ & $\begin{array}{c}0.014 \\
+0,001\end{array}$ & $\begin{array}{c}0.020 \\
+0,001\end{array}$ & $\begin{array}{c}0.017 \\
+0,002\end{array}$ \\
\hline Sulfato mg/L) & $\begin{array}{r}0.019 \\
+0,002\end{array}$ & $\begin{array}{c}0.026 \\
+0,001 \\
\end{array}$ & $\begin{array}{r}0.135 \\
+0,002\end{array}$ & $\begin{array}{c}0.14 \\
+0,001\end{array}$ & $\begin{array}{r}0.976 \\
+0,001\end{array}$ & $\begin{array}{r}0.534 \\
+0,002 \\
\end{array}$ \\
\hline Cloruro (mg/L) & $\begin{array}{r}0.10 \\
+0,010 \\
\end{array}$ & $\begin{array}{r}0.15 \\
+0,001 \\
\end{array}$ & $\begin{array}{r}0.18 \\
+0,002 \\
\end{array}$ & $\begin{array}{r}0.16 \\
+0,001 \\
\end{array}$ & $\begin{array}{c}2.07 \\
+0,006 \\
\end{array}$ & $\begin{array}{r}1.92 \\
+0,03 \\
\end{array}$ \\
\hline Dureza (mg/L) & $\begin{array}{r}24.62 \\
\pm 1,230 \\
\end{array}$ & $\begin{array}{c}31.18 \\
+0,722 \\
\end{array}$ & $\begin{array}{r}23.80 \\
+2,560 \\
\end{array}$ & $\begin{array}{r}32.01 \\
\pm 1,230 \\
\end{array}$ & $\begin{array}{r}56.63 \\
+4,440 \\
\end{array}$ & $\begin{array}{r}44.73 \\
+2,841 \\
\end{array}$ \\
\hline $\begin{array}{l}\text { Materia } \\
\text { orgánica } \\
\text { (mg/L) }\end{array}$ & $\begin{array}{c}5,28 \\
+0,507\end{array}$ & $\begin{array}{c}6.34 \\
+0,316\end{array}$ & $\begin{array}{r}10,33 \\
+0,415\end{array}$ & $\begin{array}{r}10.86 \\
+0,182\end{array}$ & $\begin{array}{r}28.41 \\
+2,061\end{array}$ & $\begin{array}{l}15.48 \\
\pm 0,31\end{array}$ \\
\hline $\begin{array}{l}\text { Sólidos } \\
\text { suspendidos } \\
\text { (mg/L) }\end{array}$ & $\begin{array}{l}6 \times 10^{-4} \\
\pm 0,00\end{array}$ & $\begin{array}{c}8.5 \times 10^{-4} \\
\pm 0,00\end{array}$ & $\begin{array}{l}2,6 \times 10^{-4} \\
\pm 0,00001\end{array}$ & $\begin{array}{l}0.002 \\
\pm 0,00\end{array}$ & $\begin{array}{c}0.011 \\
\pm 0,003\end{array}$ & $\begin{array}{l}0.004 \\
\pm 0,00\end{array}$ \\
\hline $\begin{array}{l}\text { Coliformes } \\
\text { totales } \\
\text { (NMP/100mL) }\end{array}$ & $1,6 \times 10^{3}$ & $9,2 \times 10^{2}$ & $1,6 \times 10^{3}$ & $5.9 \times 10^{4}$ & $5,4 \times 10^{7}$ & $6,8 \times 10^{4}$ \\
\hline $\begin{array}{l}\text { Coliformes } \\
\text { fecales } \\
\text { (NMP/100mL) }\end{array}$ & $5,4 \times 10^{2}$ & $1,1 \times 10^{1}$ & $9,2 \times 10^{2}$ & $1,2 \times 10^{4}$ & $2,2 \times 10^{7}$ & $6,8 \times 10^{4}$ \\
\hline
\end{tabular}

Los cloruros presentaron su máximo valor en la Estación 5 con $2.07 \mathrm{mg} / \mathrm{L}$ y el mínimo valor en la Estación 1 con $0.1 \mathrm{mg} / \mathrm{L}$, existiendo diferencias significativas entre las Estaciones 1, 2, 3 y 4 con las Estaciones 5 y 6 que presentan los mayores valores de cloruro $(p<0.05)$. La presencia de cloruros en el agua puede ser de origen natural, contaminación por aguas servidas, residuos industriales, etc. (Norma Chilena 1333 (1978). Para agua potable se encuentran dentro del rango de la Norma Chilena 409 (1984). Desde el punto de vista de su utilización en regadío, el límite máximo admisible es de 200 mg/L (NCh 1333, 1978), lo que indica que estas aguas son aptas para riego.

En relación a la dureza de las aguas, su máximo valor en la Estación 5 con $56.63 \mathrm{mg} / \mathrm{L} \mathrm{CaCO}_{3}$ y el mínimo valor en la Estación 3 con $23.80 \mathrm{mg} / \mathrm{L}$, no existiendo diferencia significativa entre las estaciones 1 y 3 y entre las estaciones 2 y $4(p<0.05)$. Los valores reportados indican que las aguas del río Traiguén son blandas y aptas para la vida acuática.

La materia orgánica en el agua del río presentó su máximo valor en la Estación 5 con 28.41 mg/L y el mínimo valor en la Estación 1 con 5.28 mg/L. La presencia de materia orgánica en las aguas servidas es una consecuencia de la actividad humana, de acuerdo con Seoanez (1995) y Barnes (1989), la presencia de materia orgánica en el agua le confiere a esta última, un elevado carácter reductor. Los 
valores de la demanda Bioquímica de Oxígeno presentaron su máximo valor en la Estación 4 con $7.06 \mathrm{mg} / \mathrm{L}$ y el mínimo valor en la Estación 2 con $1.53 \mathrm{mg} / \mathrm{L}$, existiendo diferencia significativa entre todas las Estaciones ( $p$ 0.05). De acuerdo con Seoanez (1995), la DBO permite apreciar la carga del agua en materia putrescible y su poder autodepurador, y de ello se puede deducir la carga máxima aceptable. En el muestreo realizado por la DGA en los años 1993 y 1997, en la Estación 5, la $\mathrm{DBO}_{5}$ fue en promedio más baja (2 mg/L) que la actual $(4,62 \mathrm{mg} / \mathrm{L})$, debido a que esta estación al año 2000 recibía las descargas urbanas de un 96\% de la población de la ciudad de Victoria.

Las coliformes totales medidas en el agua del río presentaron su máximo valor en la Estación 5 de $5.4 \times 10^{7}$ (NMP/100 ml) y el mínimo valor en la Estación 2 con 920 (NMP/100 ml), existiendo diferencia significativa entre todas las estaciones $(p<0.05)$. La Norma Chilena 409 (NCh 409, 1984) determina para captación de agua para consumo humano, las Coliformes totales deben ser igual a cero, luego de realizado el tratamiento, de este modo ninguna de las Estaciones estaría apta para captación de agua para consumo humano, sin antes realizar un tratamiento previo. La Estación 2 (bocatoma Empresa Servicios Sanitarios de la Araucania) donde la ciudad de Victoria capta agua para abastecer a la población de agua potable presentó un valor de 920 (NMP/100 ml) coliformes totales, siendo el valor mas bajo de todas las Estaciones. Los coliformes fecales medidas en el agua del río, presentó su máximo valor en la Estación 5 con $2.2 \times 10^{7}$ (NMP/100 ml) y el mínimo valor en la Estación 2 con 11 (NMP/100 ml), no existiendo diferencia significativa entre las Estaciones 1 y 3, al valor $p<0.05$. De acuerdo con la Norma Chilena 409 (NCh 409, 1984) los requerimientos "para captación de agua para consumo humano" deben contener cero coliformes fecales. De esta manera ningún agua del río es apta para consumo humano. En la Norma Chilena 1333 (NCh 1333, 1978) para riego, se considera un valor máximo de 1000 NMP/100 ml de Coliformes fecales. Las Estaciones 4, 5 y 6 no son aptas para riego, en especial la Estación 5 que supera en más de 22 veces a lo dispuesto en la norma. Para el agua destinada a recreación con contacto directo las coliformes fecales tienen un limite máximo de 1000 NMP/100 ml. Las Estaciones 4, 5 y 6 superan el límite de la norma.

La caracterización física y química de los ríos es de gran relevancia para establecer planes de manejo, aunque se reconoce su debilidad para registrar cambios temporales y de su capacidad integradora a nivel eco sistémico. Por ello hoy se aconseja complementar los estudios con la biota más representativa (Figueroa et al., 2007)

Las Estaciones, en relación a los diferentes usos, fueron comparadas con las Normas Chilenas, NCh 777 (1971), NCh 1333 (1978), NCh 409 (1984) y con las Normas Internacionales (Comunidad Europea (CEE), Organización Mundial de la Salud (OMS), Naciones Unidas, Environmental Protection Agency (EPA), Canadá, Francia y Brasil), de lo que se puede deducir que las aguas de ninguna de las estaciones del río Traiguén es factible de emplearse como fuente de abastecimiento para consumo humano sin tratamiento, principalmente por la presencia de coliformes totales y fecales por sobre los límites fijados por las normas.

De las 6 estaciones, y de acuerdo con la Norma Chilena 1333 (NCh 1333, 1978), sólo una puede emplearse para regadío, la estación 1. Las estaciones 1,2 , y 3 cumplirían con los requisitos necesarios para la vida acuática y para recreación con contacto directo. Al analizar la Tabla 1, se pudo ver que la Estación 2 se clasifica como agua de calidad regular, de acuerdo con la Norma Chilena (NCh 777, 1971), siendo esta estación la de captación de agua potable de la ciudad de Victoria. Las otras estaciones serían de aguas de calidad deficiente, lo que implicaría someterla a tratamiento físico - químico intensivo, si se deseara potabilizarla.

La figura 2, corresponde a un gráfico de las componentes principales 1 y 2, que en conjunto representan un 83,9 de varianza total, este plano representa las relaciones lineales entre las estaciones, los parámetros fisicoquímicos y biológicos y las componentes principales. Para su interpretación se analizan en forma conjunta las correlaciones entre las variables y componentes, así como los datos originales (Tabla 1). Se puede observar que las estaciones se ordenan en el sentido de la componente 1 , desde parte superior del río, menos contaminada a la zona media más contaminada. Las estaciones 1 y 2 de la zona alta del río presentan una mayor similitud al considerar los parámetros físico químicos como perfil de comparación, son las estaciones de mejor calidad de 
aguas, de hecho estas estaciones presentan los menores valores de coliformes, nitritos, nitratos DBO, TDS y temperatura. Las Estaciones 3 y 4 tienen una mayor similitud, presentando una menor calidad de aguas, lo que es explicado por la presencia de efluentes de una planta lechera y una curtiembre. La estación 5 corresponde a la descarga del alcantarillado y presenta los mayores valores de materia orgánica (MO), sólidos disueltos totales (TDS) cloruros, dureza y fosfatos. La estación 6 presenta menores concentraciones que la estación 5, ubicándose a 1400 metros de distancia, siendo influenciada por esta descarga encontrándose las menores concentraciones de oxígeno disuelto (1,48 mg/L) y presenta valores superiores a las estaciones 1,2,3 y 4 en sólidos disueltos, nitrógeno amoniacal, dureza, y materia orgánica.

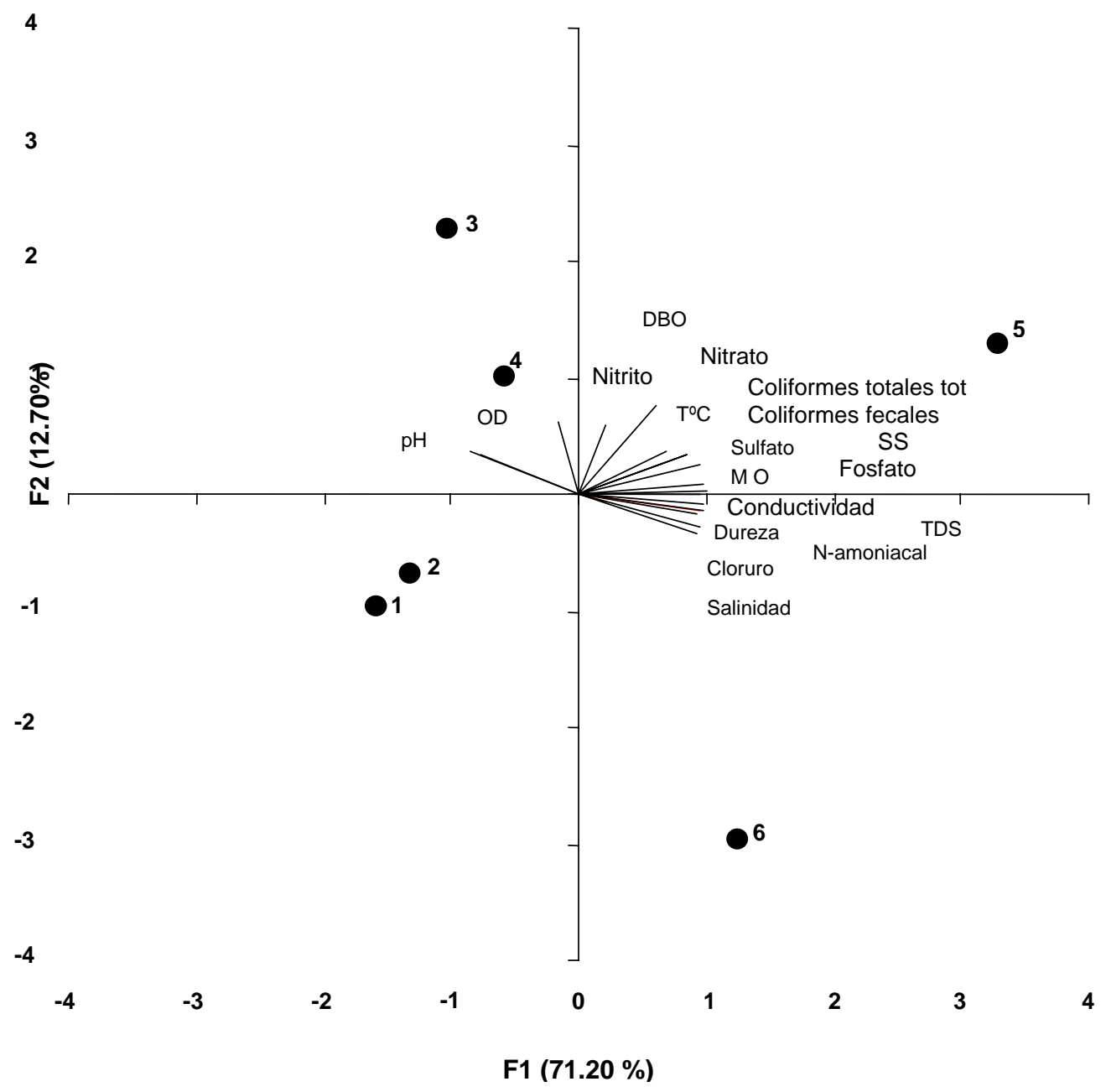

Fig. 2: Biplot de las estaciones de muestreo y parámetros físico químicos y biológicos en el plano formado por las componentes F1 y F2 del Análisis de Componentes Principales.

\section{CONCLUSIONES}

Dada la influencia de la ciudad de Victoria sobre el Río Traiguén, las estaciones de muestreo 1 y 2 ubicadas fuera del límite urbano, presentaron como tendencia general los valores mas bajos en los parámetros evaluados, en contraposición a lo que ocurre en las estaciones 4,5 y 6 donde se puede observar el impacto ocasionado por el afluente industrial en la estación 4 y el afluente urbano en la estación 5. De esta forma aparecen restricciones de uso en función de los parámetros evaluados.

Las aguas del río Traiguén de acuerdo al recuento de coliformes fecales no son aptas para consumo humano y en las estaciones 4,5 y 6 no aptas para riego, de esta forma las aguas de las estaciones 1, 2 y 3 se pueden utilizar para riego y actividad industrial, Las aguas de las estaciones 5 y 6 en cuanto al oxígeno disuelto no se encuentran dentro del rango que permite sustentar la vida acuática. 


\section{AGRADECIMIENTOS}

A la Dirección General de Investigación de la Universidad Católica de Temuco por su valiosa colaboración y al proyecto DIPUCT N²004-4-02.

\section{REFERENCIAS}

Barnes G., Tratamiento de aguas negras, 303 p., Editorial Limusa, España (1989).

CONAMA. Comisión Nacional del Medio Ambiente. Proposición de normas de calidad de aguas para proteger usos determinados. Informe final. Kristal. Chile, 180 pp. (1997).

CONAMA. Comisión Nacional del Medio Ambiente. Informe ambiental regional. Región de la Araucanía. 60 pp. (1998).

DGA, Dirección General De Aguas; Diagnóstico y clasificación de los cursos y cuerpos de agua según objetivos de calidad cuenca del río Imperial. 137 pp. (2004)

Domenech X.; Química Ambiental. El Impacto Ambiental de los Residuos. 2a Edición. Miraguano ediciones. España, 254 pp. (1994).

Field, J., K. Clarke y R. Warwick; A practical startegy analysisng multispecies distribution patttern. Mar. Ecol. Prog. Ser.: 8(3), 37-52 (1982).

Figueroa, R, y otros tres autores; Análisis comparativo de índices bióticos utilizados en la evaluación de la calidad de las aguas en un río mediterráneo de Chile: río Chillán, VIII Región. Rev. Chil. Hist. Nat. ISSN 0716-078X (en linea), 80 (2), 2007, http://www.scielo.cl/scielo_. Acceso: 08 Agosto (2008).

Gómez, A.M. y otros tres autores; Calidad del agua en la parte alta de las cuencas Juan Cojo y El Salado (Girardota - Antioquia, Colombia) Rev. Fac. Nal. Agr. Medellin. ISSN: 0304-2847 (en línea), 60 (1), 2007. http://www.scielo.org.co/scielo. Acceso: 20 de Junio (2008).

Harvey, A.; Micro-Hydro Design Manual: A Guide to small scale water power schemes. Intermediate Technology Publications London. 374 pp. (1993).

Hair J., y otros cuatro autores; Multivariate Data Análisis, Prentice Hall. 730 pp. (1998).

Herraez I. y L Rubio; Contenido en compuestos nitrogenados de las aguas subterráneas debido a los residuos urbanos. Aplicación a las terrazas cuaternarias de los ríos Jarana y Herraes. Ediciones de la Universidad Autónoma de Madrid. España, 307 pp. (1989).

INE. Censo Nacional 2002. (en línea) http://www.inearaucania.cl/banco/CENSAL/0913311_.xls. Acceso: 21de Junio (2008).

Linsley, R.; Hidrología para Ingenieros, Editorial Mc Graw-Hill, Bogotá, Colombia (1992)

MOPT Ministerio de Obras Públicas y transporte; Guía para la elaboración de estudios del medio físico. España, 800 pp. (1992).

Murgel S.; Limnología sanitaria, estudio de la polución de las aguas continentales, Monografía № 28 , Universidad de Sao Paulo, Brasil, 120 pp, (1984),

NCh, Norma Chilena Oficial № 1.333. of87 Requisitos de calidad de agua para diferentes usos. Inscripción No 49.092 por Instituto Nacional de Normalización, INN. Santiago de Chile, 20 pp. (1978).

NCh, Norma Chilena Oficial N 409/1. Agua potable - parte 1: Requisitos. Instituto Nacional de Normalización, INN. Santiago, 14 pp. (1984). 
NCh 411/6.Of98 Calidad del agua - Muestreo - Parte 6: Guía para el muestreo de ríos y cursos de agua. Instituto Nacional de Normalización, INN. Santiago, 50 pp. (1999).

NCh, Norma Chilena Oficial N777.Agua Potable Fuentes de abastecimiento y obras de captación. Terminología. Clasificación requisitos generales. Instituto Nacional de Normalización, INN. Santiago, 18 pp. (1971).

OMS., Organización Mundial de la Salud. Guías para la calidad del agua potable. Volumen 1. $3^{\circ} \mathrm{Ed}$. Ginebra 101 pp. (2004).

Seoanez M.; Ecología Industrial: Ingeniería medio ambiental aplicada a la industria y a la empresa. 522 p., Ediciones Mundi, España (1995)

Rivera N, F. Encina, A. Muñoz y P. Mejias; La calidad de las aguas en los ríos Cautín e Imperial, IX Región-Chile. Información Tecnológica: 15 (5), 89-101 (2004).

Zar, J.; Bioestistical Analysis $2^{\mathrm{a}}$ Ed. Prentice-hall, Inc. USA. 718 pp. (1984). 